\title{
ON NON-CO-HOPFIAN $p$-GROUPS WITH FINITE DERIVED SUBGROUP
}

\author{
AHMET ARIKAN \\ Gazi Üniversitesi, Gazi Eğitim Fakültesi, Matematik Eğitimi Anabilim Dal, 06500 Beşevler, \\ Ankara, Turkey \\ e-mail:arikan@gazi.edu.tr
}

(Received 6 June, 2003; accepted 27 November, 2003)

\begin{abstract}
In this article the following are proved: 1. Let $G$ be an infinite $p$-group of cardinality either $\aleph_{0}$ or greater than $2^{\aleph_{0}}$. If $G$ is center-by-finite and non- $\breve{C}$ ernikov, then it is non-co-Hopfian; that is, $G$ is isomorphic to a proper subgroup of itself. 2. Let

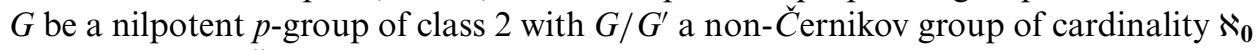
or greater than $2^{\aleph_{0}}$. If $G^{\prime}$ is of order $p$, then $G$ is non-co-Hopfian.
\end{abstract}

2000 Mathematics Subject Classification. 20E07, 20F18, $20 \mathrm{~F} 24$.

1. Introduction. A group $G$ is co-Hopfian if every injective endomorphism of $G$ is an isomorphism; that is, $G$ has no proper subgroup isomorphic to itself. Non-coHopfian groups in some classes of groups are considered by many authors. The class of abelian p-groups is well known. R. A. Beaumont and R. S. Pierce [4] showed that if the cardinality of a reduced abelian $p$-group $\mathrm{G}$ is either $\aleph_{0}$ or greater than $2^{\aleph_{0}}$, then $G$ is non-co-Hopfian. But P. Crawley [7] constructed an infinite abelian $p$-group without elements of infinite height that is co-Hopfian. Also J. M. Irwin and T. Ito [11] considered some co-Hopfian abelian $p$-groups with some additional properties. See also [1], [3], [12] for more details. In view of these results it seems natural to ask which infinite nilpotent $p$-groups are non-co-Hopfian. As a first step it seems suitable to study infinite nilpotent $p$-groups with finite derived subgroup as the results with some restrictions show in the sequel.

In [2], [5], [6] the class $\mathbf{K}$ of locally finite groups all of whose Sylow subgroups are Černikov is considered. In [6], V. V. Belyaev showed that if $G$ is a countable locally soluble K-group then $\mathrm{G}$ is co-Hopfian if and only if $G$ is hyperfinite, which was conjectured by R. Baer. This result is proved by S. D. Bell in [5] for locally finite groups independently, using character theoretic ideas. See [9] for more details.

In recent years, H. Smith and J. Wiegold [14], [15], [16] considered some non-coHopfian groups which are isomorphic to their non-nilpotent or non-abelian subgroups.

See also [8], [18] for some other results.

In this article we consider the class of center-by-finite $p$-groups (which is a subclass of the class of all locally finite $F C$-groups) and nilpotent $p$-groups of class two with derived subgroup of order $p$ and prove the following results.

THEOREM 1. Let $G$ be an infinite p-group of cardinality either $\aleph_{0}$ or greater than $2^{\aleph_{0}}$. If $G$ is center-by-finite and non-Černikov then it is non-co-Hopfian.

THEOREM 2. Let $G$ be a nilpotent p-group of class 2 with $G / G^{\prime}$ a non-Černikov group of cardinality $\aleph_{0}$ or greater than $2^{\aleph_{0}}$. If $G^{\prime}$ is of order $p$ then $G$ is non-co-Hopfian. 
2. Center-by-finite $p$-groups. We start with a Lemma which may be helpful to see the structures of the groups in some classes. Here we consider center-by-finite p-groups since these groups are always considered as an important subclass of the class of all locally finite $F C$-groups.

LEMMA 1. Let $G=F A$ be an infinite p-group with $A$ an abelian group of cardinality $\aleph_{0}$ or greather than $2^{\aleph_{0}}$ and $F$ a subgroup of $G$. If $A$ is non-ČCrnikov, $A \cap F$ is finite and $[A, F]=1$, then $G$ is non-co-Hopfian.

Proof. By Theorem 21.3 of [10] there exist radicable and reduced subgroups $D$ and $R$ respectively such that $A=D \times R$. First suppose that $D$ is a direct product of infinitely many $p^{\infty}$-type subgroups. Hence there exists a subgroup $U$ of $D$ that is a direct product of countably many $p^{\infty}$-type subgroups $A_{1}, A_{2}, \ldots$ such that $D=U \times V$ for some subgroup $V$ of $D$ and $F \cap A$ is contained in $A_{1} \ldots A_{r}$ for a natural number $r$. If $A_{i}(i=1,2, \ldots)$ is generated by $a_{i, k}$ for $k=1,2, \ldots$ then put

$$
X=R V\left\langle A_{1}, \ldots, A_{r}, A_{r+2}, \ldots\right\rangle
$$

and define $\phi$ from $A$ to $X$ by $\phi\left(a_{i, k}\right)=a_{i, k}$ for $i=1, \ldots, r$ and for all $k, \phi\left(a_{i, k}\right)=a_{i+1, k}$ for $i>r$ and $\phi(x)=x$ for all $x \in R V$. Now it is not difficult to see that $\phi$ is an isomorphism. If we define $\Phi$ from $G$ to $F X$ as $\Phi(f g)=f(\phi(g))$ for all $f \in F$ and $g \in A$ then since $[A, F]=1, \Phi$ is a homomorphism. Let $f g$ be in the kernel of $\Phi$. Then $\phi(g)=f^{-1}$ is contained in $X \cap F$ and, since $\phi$ fixes $X \cap F$ elementwise, $g$ is contained in $X \cap F$. This implies that $g=f^{-1}$; that is, $f g=1$. Hence $\Phi$ is a monomorphism. It is obvious that $\Phi$ is onto and since $F \cap A$ is contained in $X, F X$ is a proper subgroup of $G$ and thus $G$ is non-co-Hopfian.

Now suppose $D$ is a direct product of finitely many $p^{\infty}$-type subgroups. Hence $R$ must be infinite. If $R$ has finite exponent then $R$ has an infinite direct factor which is a direct product of countably many cyclic subgroups. If $R$ is countable of infinite exponent then by Exercise 8(a) on page 67 of [10] $R$ again has an infinite direct factor which is the product of countably many cyclic subgroups. If $R$ is of cardinality greater than $2^{\aleph_{0}}$ then by the proof of the Theorem (Case 3) [11, p 152] $R$ has an infinite direct factor as in the previous two cases. Hence in every case there are subgroups $M, N$ of $R$ such that $R=M \times N$ and $M$ is the direct product of countably many cyclic subgroups $\left\langle y_{i}\right\rangle$ of order $p^{k_{i}}$ such that $p^{k_{i}} \leq p^{k_{i+1}}$ for $i=1,2, \ldots$ Now there exists a natural number $n$ such that

$$
(F \cap A) \leq\left(D r_{i=1}^{n-1}\left\langle y_{i}\right\rangle\right)
$$

Put

$$
Y=N D\left\langle y_{1}, \ldots, y_{n-1}, y_{n+1}, \ldots\right\rangle
$$

and define $\theta$ from $A$ to $Y$ as $\theta\left(y_{i}\right)=y_{i}$ for $i=1, \ldots, n-1, \theta\left(y_{i}\right)=y_{i+1}^{p^{k_{i+1}-k_{i}}}$ for $i \geq n$ and $\theta(x)=x$ for all $x \in N D$. Now $\theta$ is an isomorphism. Define $\gamma$ from $G$ to $F Y$ as $\gamma(f g)=f(\theta(g))$ for all $f \in F, g \in A$. Now, $\gamma$ is an isomorphism and since $F \cap A$ is contained in $Y, F Y$ is a proper subgroup of $G$ and again $G$ is non-co-Hopfian, as desired.

Proof of Theorem 1. There is a finite subgroup $F$ such that $G=Z F$ where $Z$ is the center of $G$. Hence $G$ satisfies the hypothesis of Lemma 1 and so $G$ is non-coHopfian. 
3. Nilpotent groups of class two with derived subgroup of order $p$. Now we give a technical Lemma which concentrates on the derived factor group of a group and plays a crucial role in the proof of Theorem 2.

LeMMA 2. Let $G$ be a countably infinite nilpotent p-group of class 2 and let $K / G^{\prime}$ be a subgroup of $G / G^{\prime}$ that is a direct product of cyclic subgroups $\left\langle y_{i} G^{\prime}\right\rangle$ such that $y_{i} G^{\prime}$ is of order $p^{k_{i}}$ and $p^{k_{i}}<p^{k_{i+1}}$ for $i=1,2, \ldots$ If $G^{\prime}$ is of order $p$ then $K$ is non-co-Hopfian.

Proof. Let $z$ be a generator of $G^{\prime}$. If $y_{i}^{p_{i}}=z^{v}$ where $0<v<p$ then by considering the multiplicative inverse $l$ of $v$ in the field of elements modulo $p$ we take $y_{i}^{l}$ in place of $y_{i}$. Now $y_{i}^{p^{k_{i}}}$ can be 1 or $z$. By reordering the $y_{i}$ we have that $K=H L$ and $H \cap L=G^{\prime}$, where $H$ and $L$ are subgroups of $K$ such that if $y_{i}$ is in $H$ then $y_{i}^{p^{k_{i}}}=1$ and if it is in $L$ then $y_{i}^{p^{k_{i}}}=z$, and the $\left\langle y_{i}\right\rangle$ are ordered separately in $H$ and $L$ such that $y_{i} G^{\prime}$ is of order $p^{k_{i}}$ and $p^{k_{i}}<p^{k_{i+1}}$ for $i=1,2, \ldots$, as in the hypothesis. Clearly $H$ or $L$ is infinite. We first assume that both subgroups are infinite and show that they are non-co-Hopfian.

Every element $k$ of $H$ (and of $L$ ) can be written in the form

$$
k=\left(\Pi_{i=1}^{r} y_{i}^{t_{i}}\right) u
$$

where $0 \leq t_{i}<p^{k_{i}}, u$ is in $G^{\prime}$ and in this expression $u$ and $y_{i}^{t_{i}}$ are uniquely determined whenever $t_{i}$ is non-zero. Let $M$ denote either of $H, L$ and define $\phi$ from $M$ to $Y$ as

$$
\phi(k)=\left[\Pi_{i=1}^{r}\left(y_{i} y_{i+1}^{\left(p^{\left.k_{i+1}-k_{i}\right)}\right.} \ldots y_{i+p}^{\left(p^{k_{i+p}-k i}\right)}\right)^{t_{i}}\right] u
$$

where

$$
Y=\left\langle y_{i} y_{i+1}^{\left(p^{\left.k_{i+1}-k_{i}\right)}\right.} \ldots y_{i+p}^{\left(p^{k_{i+p}-k_{i}}\right)}, G^{\prime} \mid i=1,2, \ldots\right\rangle
$$

Since $G^{\prime}$ has order $p, G^{p}$ is contained in the center of $G$ and we have

$$
\phi(k)=\left(\Pi_{i=1}^{r} y_{i}^{t_{i}} y_{i+1}^{t_{i}\left(p^{\left.k_{i+1}-k_{i}\right)}\right.} \ldots y_{i+p}^{t_{i}\left(p^{k_{i+p}-k_{i}}\right)}\right) u .
$$

We shall show in detail that $\phi$ is an isomorphism.

Since $K / G^{\prime}$ is a direct product of cyclic groups $\left\langle y_{i} G^{\prime}\right\rangle$ (for $i=1,2, \ldots$ ), the set $\left\{y_{i} G^{\prime}: i=1, \ldots, r\right\}$ is linearly independent and thus $\phi$ is well-defined. Let

$$
l=\left(\Pi_{i=1}^{r} y_{i}^{s_{i}}\right) v
$$

where $0 \leq s_{i}<p^{k_{i}}$ and $v$ is in $G^{\prime}$. Then

$$
k l=\left(\Pi_{i=1}^{r} y_{i}^{t_{i}}\right)\left(\Pi_{i=1}^{r} y_{i}^{s_{i}}\right) u v=\Pi_{i=1}^{r}\left(y_{i}^{t_{i}+s_{i}}\right) u v w
$$

for an element $w$ of $G^{\prime}$ ( $w$ can be calculated in terms of the commutators). First assume that $\left(t_{i}+s_{i}\right)<p^{k_{i}}$ for all $i=1, \ldots, r$ and put $n_{i}=t_{i}+s_{i}$ for $i=1, \ldots, r$. Now

$$
k l=\left(\Pi_{i=1}^{r} y_{i}^{n_{i}}\right) u v w
$$

and it is in the form stated above. Thus

$$
\phi(k l)=\left[\Pi_{i=1}^{r}\left(y_{i}^{n_{i}} y_{i+1}^{n_{i}\left(p^{k_{i+1}-k_{i}}\right)} \ldots y_{i+p}^{n_{i}\left(p^{k_{i+p}-k_{i}}\right)}\right)\right] u v w,
$$


since $u v w \in G^{\prime}$ and $\phi$ fixes $u v w$ by the definition of $\phi$. We also see that

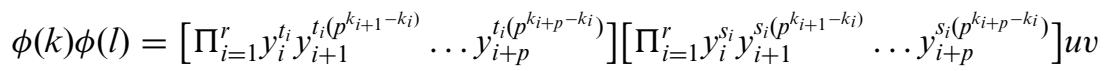

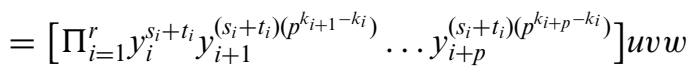

$$
\begin{aligned}
& =\left[\Pi_{i=1}^{r} y_{i}^{n_{i}} y_{i+1}^{n_{i}\left(p^{k_{i+1}-k_{i}}\right)} \ldots y_{i+p}^{n_{i}\left(p^{k_{i+p}-p_{i}}\right)}\right] u v w,
\end{aligned}
$$

since $G^{p}$ is contained in $Z(G)$. Now assume that there exist $m_{1}, \ldots, m_{c} \in\{1, \ldots, r\}$ such that $t_{m_{j}}+s_{m_{j}} \geq p^{k_{m_{j}}}$ for $j=1, \ldots, c$. Then there exist $d_{m_{j}}$ and $w_{m_{j}}$ such that $t_{m_{j}}+s_{m_{j}}=$ $p^{k_{m_{j}}} w_{m_{j}}+d_{m_{j}}$ and $0 \leq d_{m_{j}}<p^{k_{m_{j}}}$. Since $t_{m_{j}}+s_{m_{j}}<2 p^{k_{m_{j}}}$ we have $w_{m_{j}}=1$ and thus $t_{m_{j}}+s_{m_{j}}=p^{k_{m_{j}}}+d_{m_{j}}$. Define

$$
n_{i}= \begin{cases}t_{i}+s_{i} & \text { if } t_{i}+s_{i}<p^{k_{i}} \\ d_{i} & \text { if } t_{i}+s_{i} \geq p^{k_{i}}\end{cases}
$$

Now $0 \leq n_{i}<p_{k_{i}}$ for $i=1, \ldots, r$ and

$$
k l=\left(\Pi_{i=1}^{r} y_{i}^{n_{i}}\right)\left(\Pi_{j=1}^{c} y_{m_{j}}^{p^{k} m_{j}}\right) u v w .
$$

Put $g=\left(\Pi_{j=1}^{c} y_{m_{j}}^{p^{k_{m_{j}}}}\right)$. Then $g=1$ or $z^{c}$ according to whether $M$ is $H$ or $L$, respectively. It follows that

$$
\phi(k l)=\left[\Pi_{i=1}^{r} y_{i}^{n_{i}} y_{i+1}^{n_{i}\left(p^{k_{i+1}-k_{i}}\right)} \ldots y_{i+p}^{n_{i}\left(p^{k_{i+p}-k_{i}}\right)}\right] g u v w,
$$

since guvw $\in G^{\prime}$ and $\phi$ fixes guvw by the definition of $\phi$. We also have

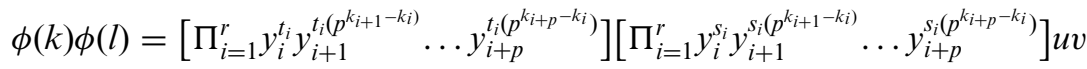

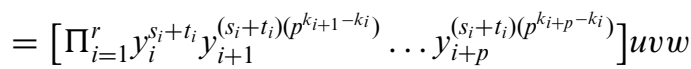

$$
\begin{aligned}
& =\left[\Pi_{i=1}^{r} y_{i}^{n_{i}} y_{i+1}^{n_{i}\left(p^{k_{i+1}-k_{i}}\right)} \ldots y_{i+p}^{n_{i}\left(p^{k_{i+p}-k_{i}}\right)}\right]\left[\Pi_{j=1}^{c} y_{m_{j}}^{p^{k_{m_{j}}}} y_{m_{j}+1}^{k_{m_{j}+1}} \ldots y_{m_{j}+p}^{p_{m_{j}+p}^{k_{m}}}\right] u v w \\
& =\left[\Pi_{i=1}^{r} y_{i}^{n_{i}} y_{i+1}^{n_{i}\left(p^{k_{i+1}-k_{i}}\right)} \ldots y_{i+p}^{n_{i}\left(p^{k_{i+p}-k_{i}}\right)}\right] g u v w,
\end{aligned}
$$

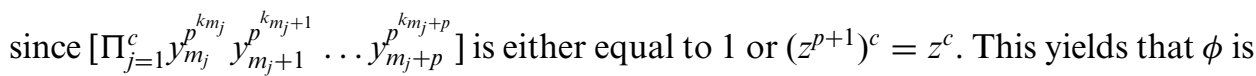
a homomorphism.

Let bars denote subgroups and elements modulo $G^{\prime}$. If $k$ is in $k e r \phi$ then we have

$$
\Pi_{i=1}^{r}\left(\bar{y}_{i}^{t_{i}} \bar{y}_{i+1}^{t_{i}\left(p^{\left.k_{i+1}-k_{i}\right)}\right.} \ldots \bar{y}_{i+p}^{t_{i}\left(p^{k_{i+p}-p_{i}}\right)}\right)=1 .
$$

Now $p^{k_{i}} \mid t_{i}$ for $i=1,2, \ldots$ and this implies that $k=1$. Consequently, we see that $\phi$ is an isomorphism and $M$ is non-co-Hopfian.

Let $y_{1}$ be an element of $Y$. Then

$$
\bar{y}_{1}=\left[\bar{y}_{1} \bar{y}_{2}^{\left(p^{k_{2}-k_{1}}\right)} \ldots \bar{y}_{1+p}^{\left(p_{p+1}^{k_{p+1}-k_{1}}\right)}\right]^{m_{1}} \ldots\left[\bar{y}_{r} \bar{y}_{r+1}^{\left(p^{k_{r+1}-k_{r}}\right)} \ldots \bar{y}_{r+p}^{\left(p^{k_{r+p}-k_{r}}\right)}\right]^{m_{r}},
$$

for some $r \geq 1$. If $r \geq 2$, then $p^{k_{r}} \mid m_{r}$, since $p^{k_{r+p}} \mid\left(p^{k_{r+p}} m_{r}\right)$. This implies that

$$
\bar{y}_{1}=\left[\bar{y}_{1} \bar{y}_{2}^{\left(p^{k_{2}-k_{1}}\right)} \ldots \bar{y}_{1+p}^{\left(p_{p+p}^{k_{p+1}-k_{1}}\right)}\right]^{m_{1}} .
$$


Now we see that $m_{1}$ is of the form $p^{k_{1}} t+1$ and $p^{k_{2}} \mid\left[\left(p^{k_{1}} t+1\right)\left(p^{k_{2}-k_{1}}\right)\right]$. But then $p^{k_{1}} \mid 1$, a contradiction.

Finally, we will show that $K=H L$ is non-co-Hopfian assuming that $H$ is infinite (if $L$ is infinite then the same method can be used). Define $\theta$ from $G$ to $Y L$ as $\theta(h l)=\phi(h) l$. Since $\phi$ fixes $G^{\prime}$ elementwise, $\theta$ is well-defined. If

$$
h=\left(\Pi_{i=1}^{r} y_{i}^{s_{i}}\right) u
$$

then

$$
l^{\phi(h)}=l^{\left(\Pi_{i=1}^{r} y_{i}^{s_{i}} y_{i+1}^{s_{i}\left(p^{\left.k_{i+1}-k_{i}\right)}\right.} \cdots y_{i+p}^{s_{i}\left(p^{k_{i+p}-k_{i}}\right)}\right) u}=l^{\left(\Pi_{i=1}^{r} y_{i}^{y_{i}}\right) u}=l^{h},
$$

since $y_{i+1}^{s_{i}\left(p^{k_{i+1}-k_{i}}\right)} \ldots y_{i+p}^{s_{i}\left(p^{k_{i+p}-k_{i}}\right)}$ is contained in the center of $G$. This shows that $\theta$ is a homomorphism. It is not difficult to see that the kernel of $\theta$ is trivial and $Y L$ is proper in $K$. Therefore $K$ is non-co-Hopfian.

Proof of Theorem 2. Case 1. $G / G^{\prime}$ is reduced. Suppose first $\operatorname{card}\left(G / G^{\prime}\right)=\aleph_{0}$ and $G / G^{\prime}$ is of infinite exponent. Then it has a direct factor $K / G^{\prime}$ which is a direct product of cyclic subgroups with strictly increasing order by Exercise 8(a) on page 67 of [10]. Now there is a subgroup $N$ of $G$ such that $G=K N$ and $N \cap K=G^{\prime}$. By Lemma 2, $K$ is non-co-Hopfian. Let $\theta$ and $\phi$ be as in the proof of Lemma 2 and let $K_{1}=\theta(K)$. Define $\gamma$ from $G$ to $K_{1} N$ as $\gamma(k n)=\theta(k) n$. Since $K=H L$ where $H$ and $L$ are as in the proof of Lemma 2,

$$
n^{\theta(k)}=n^{\theta(h l)}=n^{\phi(h) l}=n^{h l}
$$

Hence we obtain that $\gamma$ is a homomorphism. Since $K \cap N=G^{\prime}$ and $\theta$ fixes $G^{\prime}$ elementwise, ker $\gamma$ is trivial. Consequently, $\gamma$ is an isomorphism and thus $G$ is non-coHopfian.

Now let bars denote subgroups and elements modulo $G^{\prime}$ and suppose $\bar{G}$ has finite exponent. Then $\bar{G}=\bar{B}_{1} \times \ldots \times \bar{B}_{m}$ where $\bar{B}_{k}$ is a (possibly trivial) direct product of cyclic subgroups of order $p^{k}$ for $k=1, \ldots, m$ and some $\bar{B}_{j}$ is infinite. Suppose $j>1$. $\bar{B}_{j}$ has an infinite subgroup $\bar{U}$ which is a direct product of countably many cyclic subgroups $\left\langle\bar{y}_{i}\right\rangle$ of order $p^{j}$ such that $\bar{B}_{j}=\bar{U} \times \bar{V}$ for some subgroup $\bar{V}$ of $\bar{B}_{j}$. Put

$$
M=B_{1} \ldots B_{j-1} V B_{j+1} \ldots B_{m}\left\langle y_{i} y_{i+1}^{p} \mid i=1,2, \ldots\right\rangle
$$

and define $\gamma$ from $G$ to $M$ as $\gamma\left(y_{i}\right)=y_{i} y_{i+1}^{p}$ for $i=1,2, \ldots$ and $\gamma(x)=x$ for all $x \in B_{1} \ldots B_{j-1} V B_{j+1} \ldots B_{m}$. Using an argument as in the proof of Lemma 2 and the fact that $y_{j+1}^{p^{j+1}}=1$ we can show that $\gamma$ is an isomorphism and $M \neq G$. Hence $G$ is nonco-Hopfian. If $j=1$, then $\bar{B}_{1}$ is an infinite elementary abelian $p$-group. Now $\bar{B}_{1}$ has a direct factor $\bar{H}$ which is a direct product of countably many cyclic subgroups such that $\bar{B}_{1}=\bar{H} \times \bar{L}$ for a subgroup $\bar{L}$ of $\bar{G}$. Put $G^{\prime}=\langle z\rangle$ and define $\phi: \bar{H} \times \bar{H} \rightarrow G F(p)$ as $\phi(\bar{a}, \bar{b})=k$ where $[a, b]=z^{k}$. It is easy to show that $\phi$ is a non-degenerate, alternating bilinear form. Since $\operatorname{dim}_{F}(\bar{H})$ is countable where $F=G F(p), \bar{H}$ is an orthogonal direct product of hyperbolic planes $\bar{H}_{1}, \bar{H}_{2} \ldots$ If we consider the pre-image of each one of these hyperbolic planes then we can find $a_{i}, b_{i}$ for $i=1,2, \ldots$ such that $H=\left\langle a_{1}, b_{1}\right\rangle\left\langle a_{2}, b_{2}\right\rangle \ldots$ where $\left[a_{1}, b_{1}\right]=\left[a_{2}, b_{2}\right]=\ldots$ and $\left[\left\langle a_{i}, b_{i}\right\rangle,\left\langle a_{j}, b_{j}\right\rangle\right]=1$ if $i \neq j$. Now define $\gamma$ from $G$ to $Y$ as $\gamma\left(a_{i}\right)=a_{i+1}, \gamma\left(b_{i}\right)=b_{i+1}$ and $\gamma(x)=x$ for all $x \in L$ 
where

$$
Y=L\left\langle a_{i}, b_{i} \mid i=2,3, \ldots\right\rangle .
$$

Then $\gamma$ is an isomorphism, $Y \neq G$ and thus $G$ is non-co-Hopfian.

Finally suppose $\bar{G}$ is of infinite exponent and cardinality greater than $2^{\aleph_{0}}$. Then

$$
\bar{G}=\bar{B}_{1} \times \ldots \times \bar{B}_{j} \times \bar{G}_{j}
$$

for a subgroup $\bar{G}_{j}$ of $\bar{G}$. By the proof of the Theorem (Case 3) on page 152 of [11] some $\bar{B}_{j}$ must be infinite. Arguing the same way as in the previous case we see that $G$ is non-co-Hopfian for $j=1$ and for $j>1$ with few changes. If $\bar{G}$ is of finite exponent then again the above arguments work.

Case 2. $G / G^{\prime}$ is not reduced. Now if $D / G^{\prime}$ is the radicable part of $G / G^{\prime}$ then $G / G^{\prime}=D / G^{\prime} \times R / G^{\prime}$ for a reduced subgroup $R / G^{\prime}$ of $G / G^{\prime}$ by Theorem 21.3 of [10]. If $D$ is non-abelian then $D^{\prime}=G^{\prime}$ and hence $D$ is radicable by Theorem 9.23 of [13]. This implies that $D$ is abelian by Corollary 2 to Theorem 9.23 of [13], a contradiction. If $D$ is not radicable then $D=G^{\prime} \times D^{p}$ and thus $D^{p}$ is radicable. Put $A=D^{p}$ then $G=A R$ and $A$ centralizes $R$. Hence $R$ cannot be abelian, since $G$ is nilpotent of class two. Now $\operatorname{card}(R)$ or $\operatorname{card}(A)$ is either $\aleph_{0}$ or greater than $2^{\aleph_{0}}$; otherwise $\operatorname{card}\left(G / G^{\prime}\right)$ would equal $2^{\aleph_{0}}$. If $\operatorname{card}(A)$ has that property, then $G$ is non-co-Hopfian by Lemma 1 . Hence we may assume that $\operatorname{card}(R)$ has the above property. If we define $\Phi$ as $\Phi(a r)=a \gamma(r)$ for all $a \in A$ and $r \in R$ then $\Phi$ is an isomorphism and thus $G$ is non-co-Hopfian. This completes the proof.

AcKnowledgment. The author is grateful to Professor Martyn Russell Dixon for many helpful discussions and to the referee for valuable comments and suggestions.

\section{REFERENCES}

1. R. Baer, Groups without proper isomorphic quotient groups, Bull. Amer. Math. Soc. 51 (1944), 267-277.

2. R. Baer, Lokal endlich-auflösbare Gruppen mit endlichen Sylowuntergruppen, J. Reine Angew. Math 239/240 (1970), 109-144.

3. R. A. Beaumont, Groups with isomorphic proper subgroups, Bull. Amer. Math. Soc. 51 (1945), 381-387.

4. R. A. Beaumont and R. S. Pierce, Partly transitive modules and modules with proper isomorphic submodules, Trans. Amer. Math. Soc. 91 (1959), 209-219.

5. S. D. Bell, Locally finite groups with Černikov Sylow subgroups, $\mathrm{PhD}$ thesis (University of Manchester, 1989). $1-11$.

6. V. V. Belyaev, Locally inner endomorphisms of $S F$-groups, Algebra and Logic 27 (1988),

7. P. Crawley, An infinite primary abelian p-group without proper isomorphic subgroups, Bull. Amer. Math. Soc. 68 (1962), 463-467.

8. S. Deo and K. Varadarajan, Hopfian and co-Hopfian groups, Bull. Austral. Math. Soc. 56 (1997), 17-24.

9. M. R. Dixon, Sylow theory, formations and fitting classes (World Scientific, 1994).

10. L. Fuchs, Infinite abelian groups, Vols. 1 and 2 (Academic Press, 1973).

11. J. M. Irwin and T. Ito, A quasi-decomposable abelian group without proper isomorphic quotient groups, Pacific J. Math. 29 (1969), 151-160.

12. I. Kaplansky, A note on groups without isomorphic subgroups, Bull. Amer. Math. Soc. 51 (1945), 529-530. 
13. D. J. S. Robinson, Finiteness conditions and generalized soluble groups, Vols. 1 and 2 (Springer-Verlag, 1972).

14. H. Smith and J. Wiegold, Groups which are isomorphic to their nonabelian subgroups, Rend. Sem. Mat. Univ. Padova 97 (1997), 7-16.

15. H. Smith and J. Wiegold, Groups isomorphic to their non-nilpotent subgroups, Glasgow Math. J., 40 (1998), 257-262.

16. H. Smith and J. Wiegold, Soluble groups isomorphic to their non-nilpotent subgroups, J. Austral. Math. Soc. Ser.A, 67 (1999), 399-411.

17. M. J. Tomkinson, FC-groups, Research Notes in Mathematics (Pitman, 1984).

18. P. J. Witbooi, Groups for which every subquotient is (co)-Hopfian, New Zealand J. Math. 26 (1997), 301-308. 